Technical note

\title{
Exact expressions for numerical evaluation of high order modes of vibration in uniform Euler-Bernoulli beams
}

\author{
Paulo J. Paupitz Gonçalves ${ }^{\mathrm{a}, *}$, Andrew Peplow ${ }^{\mathrm{b}}$, Michael J. Brennan ${ }^{\mathrm{c}}$ \\ a São Paulo State University - UNESP, School of Engineering, Bauru, Brazil \\ b Zayed University, United Arab Emirates \\ c São Paulo State University - UNESP, School of Engineering, Ilha Solteira, Brazil
}

\section{A B S T R A C T}

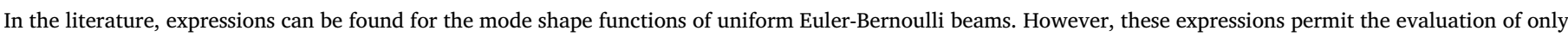

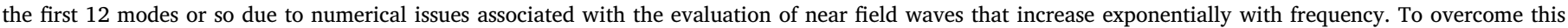

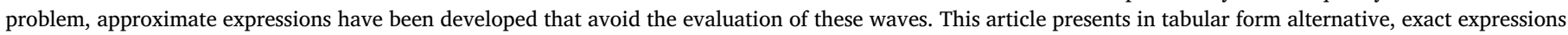

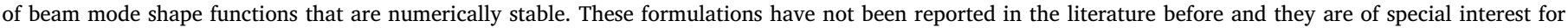

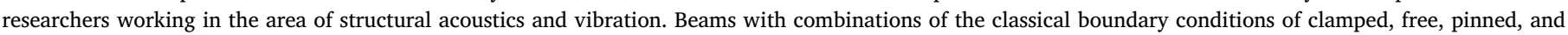
sliding are considered.

\section{Introduction}

Mode shape functions often need to be evaluated numerically when calculating the forced response of a structure. In many cases, only a few modes of vibration needs to be considered and common forms of modeshape functions found in textbooks, for example [1-6] are suitable. However, in some cases many modes need to be considered. Common forms of expressions for the mode shape functions found in the literature may not be suitable, as they permit the evaluation of only the first 12 modes or so due to numerical issues that arise from the evaluation of nearfield waves. This has been acknowledged by researchers, and some methods have been proposed to overcome the problem. In the study of the vibrations of a truss structure, Shankar and Keane [7] reformulated the expression for the mode shape of a free-free beam so that it could be numerically evaluated up to the first two hundredth mode or so. Using beam mode shape functions, Warburton [8], gave mode shape expressions for transverse plate vibration for some boundary conditions that are numerically stable up to a certain value of $k l$ in which $k$ is the wavenumber and $l$ is the length of the beam. For stepped-beams [9], the authors introduce a local coordinate system to overcome possible round-off errors in computing modes in the global coordinate system. However, the principle of the method will start to breakdown once round-off errors in any of the local coordinate systems appear. Tang [10] has also given expressions for beam mode shape functions with some other boundary conditions, but again these are only numerically stable up to a certain value of $k l$. To overcome this problem Gonçalves et al. [11] made some approximations for $k l \gg 1$ and presented numerically stable alternative approximate expressions for generally available mode shape functions. This introduced small errors which are larger for small values of $k l$, but converge for $k l \gg 1$. The purpose of this technical brief is to present, in tabular form, alternative exact expressions for uniform beam mode shape functions that do not suffer from numerical ill-conditioning for any value of $k l$, and are not currently available in the literature. This is in contrast to the work in Ref. [11] which reported approximate rather than exact expressions. It should be noted that the exact expressions are only valid up to frequencies, where the Euler-Bernoulli beam theory is valid, that is when the thickness of the beam is less than about one tenth of a wavelength.

\section{Problem statement}

To illustrate the problem of numerical ill-conditioning, the expression for a clamped-clamped beam mode shape function for a uniform beam of density $\rho$, Young's modulus $E$, cross-sectional area $S$, second moment of area $I$, and length $l$ given by $[1,3]$

$\phi_{n}=\cosh \left(k_{n} x\right)-\cos \left(k_{n} x\right)-\sigma_{n}\left(\sinh \left(k_{n} x\right)-\sin \left(k_{n} x\right)\right)$

is considered. In Eq. (1), $k=\left(\frac{\rho S}{E I}\right)^{1 / 4} \omega^{1 / 2}$ is the bending wavenumber, which is a function of angular frequency $\omega$ and

$\sigma_{n}=\frac{\cosh \left(k_{n} l\right)-\cos \left(k_{n} l\right)}{\sinh \left(k_{n} l\right)-\sin \left(k_{n} l\right)}$

\footnotetext{
* Corresponding author.

E-mail addresses: paulo.jpg@feb.unesp.br (P.J.P. Gonçalves), andrew.peplow@zu.ac.ae (A. Peplow), mj.brennan@unesp.br (M.J. Brennan).
} 


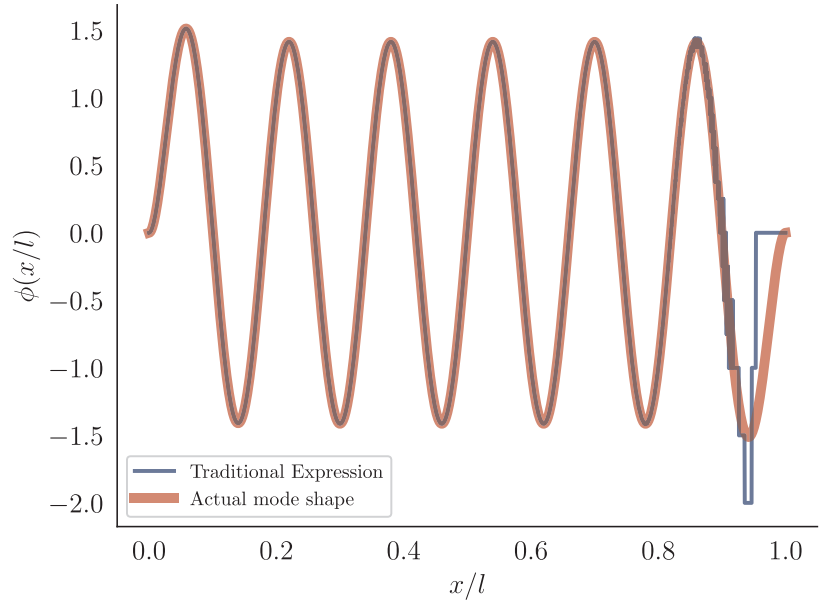

Fig. 1. Comparison of the actual mode shape and the mode shape calculated using the traditional expressions found in textbooks.

where the subscript $n$ denotes the $n$-th mode of vibration. The mode shape for $n=12$ is shown in Fig. 1. It can be seen that the near field wave close to the end where $x / l=1$ is not correctly calculated. To overcome this problem Eqs. (1) and (2) need to be reformulated. In general, the numerical evaluation of some functions such as $e^{-x}-1, \operatorname{Acosh}(x)-\operatorname{Bsinh}(x)$, can lead to round-off errors using floating point arithmetic [12]. Some of these functions can be rewritten, for example $\cosh (x)-\sinh (x)=e^{-x}$, which is a better form for numerical evaluation by a computer. For cases similar to $e^{-x}-1$, some computer languages have algorithms to reduce the round-off errors [13]. In Ma$\mathrm{tlab}^{\circledR}$ for instance, $\exp (\mathrm{x})-1$ can be computed using the function $\operatorname{expm1}(\mathrm{x})$.

\section{Numerically stable mode shape expressions}

It is possible to rewrite any of the beam mode shape functions in a format such that the round-off errors are negligibly small. This is illustrated here for a clamped-clamped beam, but the method can be applied to any mode shape function. To obtain an expression for the mode shape that is numerically stable, Eq. (2) can be modified by multiplying both its numerator and denominator by $e^{-k_{n} l}$, so that

$\sigma_{n}=\frac{1+e^{-2 k_{n} l}-2 \cos \left(k_{n} l\right) e^{-k_{n} l}}{1-e^{-2 k_{n} l}-2 \sin \left(k_{n} l\right) e^{-k_{n} l}}$

Note that $\sigma_{n}$ tends to 1 as $k_{n}$ increases, so Eq. (3) will not have numerical issues for high frequencies. Similarly, Eq. (1), can also be modified writing the hyperbolic functions in terms of exponentials to give,

$\phi_{n}=\frac{e^{k_{n} x}}{2}\left(1-\sigma_{n}\right)+\frac{e^{-k_{n} x}}{2}\left(1+\sigma_{n}\right)-\cos \left(k_{n} x\right)+\sigma_{n} \sin \left(k_{n} x\right)$

The only term in Eq. (4) that needs to be reformulated is $e^{k_{n} x}\left(1-\sigma_{n}\right) / 2$. This is done by substituting for $\sigma_{n}$ from Eq. (3) and expanding to give

$\frac{e^{k_{n} x}}{2}\left(1-\sigma_{n}\right)=\frac{-e^{k_{n}(x-2 l)}+\left(\cos \left(k_{n} l\right)-\sin \left(k_{n} l\right)\right) e^{k_{n}(x-l)}}{1-e^{-2 k_{n} l}-2 \sin (k l) e^{-k_{n} l}}$

Finally, Eqs. (4) and (5) are combined to give a mode shape expression that is numerically stable and is exact for all values of $k_{n} l$, which is

$\begin{aligned} \phi_{n}= & \frac{-e^{k_{n}(x-2 l)}+\left(\cos \left(k_{n} l\right)-\sin \left(k_{n} l\right)\right) e^{k_{n}(x-l)}}{1-e^{-2 k_{n} l}-2 \sin (k l) e^{-k_{n} l}}+\frac{1}{2} e^{-k_{n} x}\left(1+\sigma_{n}\right)-\cos \left(k_{n} x\right) \\ & +\sigma_{n} \sin \left(k_{n} x\right)\end{aligned}$

This is given in Table 1 for ease of reference. Numerically stable and exact expressions for the mode shape functions are also given in Table 1 for the most common boundary conditions.

To obtain the wavenumber $k_{n}$ and hence the $n$-th natural frequency,

Table 1

Bending mode shapes expressions for different boundary conditions.

\begin{tabular}{|c|c|c|}
\hline $\begin{array}{lr}\mid l & l \\
0 & \text { clamped-clamped }\end{array}$ & $\phi_{n}(x)=\left[\frac{-e_{x 2 l}+\left(c_{l}-s_{l}\right) e_{x l}}{1-e_{2 l}-2 s_{l} e_{l}}\right.$ & $+\frac{1}{2} e_{x}\left(1+\sigma_{n}\right)-c_{x}+\sigma_{n} s_{x}$ \\
\hline $0 \quad$ free-free $\quad l$ & $\phi_{n}(x)=\left[\frac{-e_{x 2 l}+\left(c_{l}-s_{l}\right) e_{x l}}{1-e_{2 l}-2 s_{l} e_{l}}\right.$ & $+\frac{1}{2} e_{x}\left(1+\sigma_{n}\right)+c_{x}-\sigma_{n} s_{x}$ \\
\hline
\end{tabular}

$k_{1} l=4.73004$

$k_{2} l=7.85320$

$k_{3} l=10.9956$

$k_{4} l=14.1372$

$k_{5} l=17.2788$

$k_{n} l=\frac{(2 n+1) \pi}{2}$, for $n>5$

$k_{1} l=1.87510$

$k_{2} l=4.69409$

$k_{3} l=7.85476$

$k_{4} l=10.9955$

$k_{5} l=14.1372$

$k_{n} l=\frac{(2 n-1) \pi}{2}$, for $n>5$

$k_{1} l=3.92660$

$k_{2} l=7.06858$

$k_{3} l=10.2102$

$k_{4} l=13.3518$

$k_{5} l=16.4934$

$k_{n} l=\frac{(4 n+1) \pi}{4}$, for $n>5$

$k_{1} l=2.36502$

$k_{2} l=5.49780$

$k_{3} l=8.63938$

$k_{4} l=11.7810$

$k_{5} l=14.9226$

$k_{n} l=\frac{(4 n-1) \pi}{4}$,

for $n>5$

* System with rigid body modes $e_{x 2 l}=e^{k_{n}(x-2 l)}, e_{x l}=e^{k_{n}(x-l)}, e_{x}=e^{-k_{n} x}, e_{l}=e^{-k_{n} l}, e_{2 l}=e^{-2 k_{n} l}, c_{x}=\cos \left(k_{n} x\right), s_{x}=\sin \left(k_{n} x\right), c_{l}=\cos \left(k_{n} l\right), s_{l}=\sin \left(k_{n} l\right)$. 
a numerically stable expression should be used. For example, the wavenumbers for a clamped-clamped beam may be obtained by multiplying the fundamental characteristic frequency equation found in many textbooks, for example [1-3], by $2 e^{-k l}$, i.e.

$F(k)=\cos (k l) \cosh (k l)-1 \Rightarrow \cos (k l)\left(1+e^{-2 k l}\right)-2 e^{-k l}$

where $F(k)$ is the frequency equation, in which $k$ is dependent on the boundary conditions. This change avoids cancellation errors that can occur in the numerical solver such as MATLAB's fsolve.

\section{Conclusions}

This article has discussed the problem of evaluating beam mode shape functions of a beam when a wavelength is much smaller than the length of the beam. Alternative expressions that do not suffer from numerical ill-conditioning have been presented and have been tabulated for ease of reference.

\section{Appendix A. Supplementary data}

Supplementary data associated with this article can be found, in the online version, at https://doi.org/10.1016/j.apacoust.2018.05.014.

\section{References}

[1] Bishop RED, Johnson DB. The Mechanics of Vibration. Cambridge: Cambridge Press; 1960.

[2] Rao SS. Mechanical vibrations. 6th ed. Pearson; 2016.

[3] Rao SSS. Vibration of continuous systems. 1st ed. Wiley; 2007.

[4] Leissa AW, Qatu MS. Vibration of continuous systems. McGraw-Hill Education; 2011.

[5] Erturk A, Inman DJ. Piezoelectric energy harvesting. Wiley; 2011.

[6] Gardonio P, Brennan MJ. Chapter 9 in Advanced applications in acoustics, noise and vibration. Spon Press Publisher; 2004.

[7] Shankar K, Keane AJ. Energy flow predictions in a structure of rigidly joined beams using receptance theory. J Sound Vib 1995;185(5):867-90.

[8] Warburton GB. The Vibration of Rectangular Plates. Proc Inst Mech Eng 1954;168:371-84.

[9] Xu W, Cao M, Ren Q, Su Z. Numerical evaluation of high-order modes for stepped beam. J Vib Acoust 2013;136:14503.

[10] Tang Y. Numerical evaluation of uniform beam modes. J Eng Mech ASCE 2003;129:1475-7.

[11] Goncalves P, Brennan M, Elliott S. Numerical evaluation of high-order modes of vibration in uniform Euler-Bernoulli beams. J Sound Vib 2007:301(3-5):1035-9.

[12] IEEE Standards Committee, 754-2008 - IEEE Standard for Floating-Point Arithmetic, (n.d.)

[13] N.H.F. Beebe, Computation of $\operatorname{expm} 1=\exp (\mathrm{x})-1$, 2017. http://www.math.utah. edu/ beebe/reports/expm1.pdf. 